\title{
Signal, noise, and detective quantum efficiency of a-Si:H flat-panel imagers
}

Jeffrey H. Siewerdsen

Department of Radiation Oncology, University of Michigan, Ann Arbor, Michigan 48109-0010

[E-mail: jsiewerd@beaumont.edu, Fax: 248-551-0089]

Thesis Advisors: Larry E. Antonuk, Ph.D. and Michael J. Longo, Ph.D.

Ph.D. awarded June 1998 by The Department of Physics, University of Michigan, Ann Arbor, Michigan

Flat-panel imagers based upon the technology of thin-film amorphous silicon transistors and photodiodes are under investigation for a wide variety of medical imaging applications. This thesis presents quantitative empirical and theoretical investigations of the imaging performance of such imaging systems. Performance was evaluated in terms of imager signal size, spatial resolution, noise characteristics, and signal-to-noise ratio for a wide variety of imaging system configurations and exposure conditions relevant to medical imaging. A theoretical model based upon cascaded systems analysis allowed prediction of imager signal, noise, and detective quantum efficiency (DQE), and theoretical results were found to agree well with empirical measurements. The empirical and theoretical analyses yielded quantification of the performance of existing imager designs, allowed investigation of the potential performance of future flat-panel imaging systems, and provided a methodology for identifying optimal imager configurations for various applications and imaging tasks. There is every indication that flat-panel imagers could provide performance superior to that of existing clinical imaging technologies. For example, in general x-ray radiography, mammography, and radiotherapy portal imaging, such systems could provide DQE exceeding $60 \%$, $80 \%$, and $1.5 \%$, respectively, approximately twice that of film-based systems. However, for applications involving very low exposures per image, e.g., real-time fluoroscopy, flat-panel imagers may suffer from reduced signal-to-noise ratio. The analyses developed in this thesis provide a general description of imager signal and noise as well as a means of identifying strategies for improved imager performance.

[Copies of the thesis are available from UMI Dissertation Services, 300 N. Zeeb Rd. Ann Arbor, MI 48106-1346; Phone: 800-521-0600; Fax: 734-761-7201.] 